\title{
MUJERES RURALES PROFESIONALES: SU EVALUACIÓN DEL MEDIO RURAL EN CATALUÑA Y GALICIA ${ }^{1,2}$
}

\author{
Maria Dolors Garcia-Ramon \\ Universitat Autònoma de Barcelona \\ Mireia Baylina \\ Universitat Autònoma de Barcelona \\ Ana María Porto \\ Universidad de Santiago de Compostela \\ Isabel Salamaña \\ Universitat de Girona \\ Montserrat Villarino \\ Universidad de Santiago de Compostela
}

Resumen: Dentro del contexto de las nuevas ruralidades algunas mujeres profesionales han decidido quedarse en el mundo rural para desarrollar su propio proyecto profesional y vital. Es un fenómeno nuevo dado el proceso constante de emigración rural hacia las ciudades que España ha sufrido en el pasado. La investigación la hemos llevado a cabo en zonas rurales de Cataluña y Galicia a través de entrevistas en profundidad. Nuestro objetivo es captar cómo estas mujeres profesionales perciben no solo el mundo rural sino también a las mujeres rurales actuales. Su percepción, fruto de un proceso de formación y experiencia de vida en otros lugares, urbanos, se puede convertir en un espejo para las generaciones más jóvenes de mujeres.

Palabras clave: Mujeres profesionales, medio rural, Cataluña, Galicia.

\section{Professional rural women: their evaluation of rural areas in Catalonia and Galicia}

Abstract: Within the context of the new ruralities reshaped by globalization processes some educated women decide to stay at the countryside where they develop their own life project. It is a relatively new phenomenon taking into account the steady process of female migration from rural areas that has taken place in Spain for decades. Through in-depth interviews we attempt to set out how rural women perceive themselves and assess rurality nowadays in rural areas of two Spanish regions, Catalonia and Galicia. Women's critical perceptions of the new ruralities, created by a process of education and urban life experiences, may become a mirror for younger (female) generations.

Key words: Professional women, rural areas, Catalonia, Galicia.

Data de recepció: 5 de novembre de 2014 / Data d'acceptació: 3 de desembre de 2014.

${ }^{1}$ Esta investigación forma parte de un proyecto más amplio sobre Mujeres, trabajo y ruralidad: estrategias innovadoras para el desarrollo de un proyecto profesional y personal (Ref. 2011 a 0004-INV00024), financiado por el Instituto de la Mujer, Ministerio de Sanidad, Política Social e Igualdad del Gobierno de España.

${ }^{2}$ La primera firmante de este artículo, catedrática de Geografía Humana de la Universidad Autónoma de Barcelona, ha querido participar con esta contribución en reconocimiento al catedrático Eugenio Burriel en su jubilación. Al profesor Burriel le desea agradecer la labor que desarrolló durante su estancia en el Departamento de Geografía de la UAB (de 1977 a 1980), años en que contribuyó decisivamente a abrir horizontes académicos, dejando un cálido recuerdo en quienes entonces formábamos parte del mismo. 


\section{INTRODUCCIÓN}

La mayor parte de la bibliografía sobre la sociedad rural en España ha argumentado que las mujeres son esenciales para la sostenibilidad social y económica de las zonas rurales (Camarero et al., 1991; Sabaté, 1992; Garcia Ramon et al., 1994; Garcia Ramon y Baylina, 2000; Little, 2001; Camarero, 2009; Sampedro, 2009; MARM, 2011). Sin embargo, desde hace varias décadas, se está produciendo un proceso constante de despoblación rural selectiva en España en relación con el género y la edad, que afecta especialmente a las mujeres jóvenes (Fademur, 2009), como ya ha sucedido en otros contextos europeos (Sachs, 1996; Buller y Hoggart, 2004; Goverde et al., 2004). A finales del siglo XX, esta tendencia está cambiando poco a poco y se ha observado una ligera recuperación demográfica después de décadas de despoblamiento, emigración y envejecimiento demográfico desigual entre regiones. La inmigración, las nuevas iniciativas económicas, el desarrollo de infraestructuras y servicios pueden explicar en cierta medida la vitalidad en determinadas zonas, que son las que tienen una mayor densidad de población, y mejores infraestructuras y comunicaciones (Hoggart y Paniagua, 2002; García y Sánchez, 2005; Morén y Solana, 2006; Guirado, 2010; Bayona y Gil, 2013). En este contexto, algunas mujeres con formación y profesionales han decidido quedarse o incluso irse al campo, donde quieren desarrollar su propio proyecto de vida (Carbó et al., 2013). Este es un nuevo fenómeno de interés que podría producirse en otras zonas rurales de Europa con condiciones similares.

Por lo tanto, las mujeres con educación se construyen a sí mismas como mujeres rurales y construyen la ruralidad actual. Los vínculos que establecen las mujeres con el lugar, sus experiencias y la reflexión sobre su entorno son fundamentales en la construcción de su propia identidad, para imaginar su estancia en el medio rural, y por la importancia de los valores que pueden transmitir a las generaciones más jóvenes.

Nuestro objetivo es analizar qué piensan estas mujeres rurales profesionales sobre "el medio rural" en la actualidad y cómo describen la "mujer rural" de hoy, y si se identifican o no con esta idea. Las mujeres en sus discursos sobre la ruralidad, describen e informan sobre la vida cotidiana de la población rural desde una clara perspectiva de género (Baylina \& Berg, 2010). Su valor radica en ofrecer el significado en primera persona de la vida rural de un grupo social informado y reflexivo, al que no siempre se reconoce (Halfacree, 2006, 2007). Sus percepciones de la ruralidad y de sí mismas como "mujeres rurales" constituyen un documento importante que nos ofrece mucha información acerca de la vida cotidiana, la agencia, el conocimiento y el futuro, ya que sus pensamientos pueden influir en las actitudes y prácticas de las generaciones femeninas más jóvenes.

Las mujeres han aprendido a vivir en el medio rural con el recuerdo de sus propias vivencias o las de sus antecesores/as y con la formación y la experiencia que les ha proporcionado la vida en otros lugares, urbanos. Este proceso de aprendizaje ha ido en paralelo a la construcción de un nuevo sentimiento de arraigo, que ha sido imprescindible para reafirmar su decisión de vivir en el medio rural y construir una nueva identidad. En sus apreciaciones sobre el medio rural y la mujer rural actuales se reflejan esta formación y vivencias específicas que probablemente no se encontrarían en otras mujeres y hombres de los mismos lugares.

En la primera sección de este artículo se introduce brevemente la metodología y las áreas de estudio. En las secciones segunda y tercera se aborda el análisis de "qué es el medio rural" y "qué es una mujer rural" de acuerdo con las mujeres entrevistadas. La última sección presenta unas reflexiones finales. 


\section{METODOLOGÍA Y ÁREAS DE ESTUDIO}

El estudio se ha realizado en base a una metodología cualitativa y etnográfica a partir de entrevistas en profundidad realizadas a cuarenta mujeres de zonas rurales de las comarcas de Alt Urgell, Baix Empordà y la Conca de Barberà, en Cataluña y A Ulloa, O Morrazo y O Sar, en Galicia, que han decidido desarrollar su proyecto profesional y vital en el medio rural. La mayoría de mujeres han nacido en la zona, aunque muchas de ellas han vivido en ciudades durante un tiempo, otras han nacido en el medio urbano pero tienen vínculos familiares en el medio rural y otras proceden de zonas urbanas sin vínculos directos con este medio. Se trata de mujeres de entre 30 y 50 años de edad, con estudios universitarios, casadas o con pareja heterosexual y mayoritariamente con hijos. Algo más de la mitad de las mujeres son gerentes de su propia empresa o autónomas y la mayoría trabajan en el sector servicios seguido del sector agrario.

Las entrevistas abordan muchos aspectos sobre los espacios y tiempos de su vida cotidiana con especial atención a la esfera del trabajo en sentido amplio. Se pregunta sobre su proceso de formación, el trabajo productivo, doméstico y de cuidado, el ocio y las relaciones sociales, la salud y el bienestar y la vida cotidiana y el futuro de sus hijos e hijas. Paralelamente, también se indaga en profundidad sobre su percepción del medio rural, sobre los recursos y potencialidades de la zona rural en que viven, sobre el impacto cotidiano de la crisis económica, sobre la situación particular de las mujeres en este medio y sobre los avances y retos pendientes en materia de igualdad de géneros. Una parte significativa del guión a las mujeres ha sido utilizado para las entrevistas a siete hombres profesionales de las mismas zonas a fin de comparar la versión masculina de la población rural en la actualidad y evaluar la posición relativa de las mujeres.

Las entrevistas, de dos horas de duración cada una, han sido realizadas en el domicilio de la entrevistada, en su lugar de trabajo o en el lugar que la persona ha escogido para ello, y han sido grabadas, transcritas y codificadas para su posterior análisis. En este artículo se ha trabajado solamente con algunos de los aspectos tratados en las conversaciones, principalmente los referidos a la idea y percepción del medio rural y de la mujer rural actual, la evaluación de las transformaciones socioeconómicas recientes y la forma de construir su arraigo en el medio rural.

Cataluña y Galicia son dos regiones con características distintas en España. Cataluña (7,5 millones de habitantes) tiene un PIB de $26.516 €$, muy por encima de la media en España $(22.819 €)$, y actúa como uno de los motores económicos del país. Galicia (2,7 millones de habitantes) tiene un PIB de $20.442 €$, tradicionalmente, ha sido una región de fuerte emigración y ahora es una región de servicios donde la agricultura sigue siendo relativamente importante para el empleo y los ingresos. El porcentaje de población rural en ambas áreas es también diferente (31\% en Galicia, 19\% en Cataluña), aunque de 1996 a 2009 se ha observado un aumento del 11,8\% en Cataluña y un descenso del -7,3\% en Galicia. El porcentaje de población rural es también distinto (31\% en Galicia y 19\% en Cataluña, donde sólo el $1 \%$ es población agrícola activa). Es interesante observar que entre 1996 y 2009 se produjo un aumento de la población rural en Cataluña (+12\%) y un descenso $(-7,3 \%)$ en Galicia. Este aumento de la población rural en Cataluña se debe a un proceso gradual de re-ruralización (de la población autóctona e inmigrante), que está más relacionado con la mejora de las infraestructuras, servicios y equipamientos que con una 
recuperación de la actividad agrícola (emplea sólo el 1\% de la población de Cataluña) (IDESCAT, 2012). Este cambio no se ha producido en Galicia, donde continúa la disminución constante de población rural.

\section{3. ¿QUÉ ES EL MEDIO RURAL?}

Las mujeres reconocen el concepto de rural pero consideran muy difícil construir una definición tal y como también señalan los especialistas en ciencias sociales (Aldomà, 2009; García, 2011; Woods, 2011). A veces, su primera respuesta es una pregunta para nosotras, para la entrevistadora: "Pero, cuando dices rural, ¿a qué te refieres?".

\section{a) Dicotomía rural-urbano y diferencias intra-rurales}

Algunos indicadores espaciales siguen siendo importantes para definir el medio rural entre muchas mujeres:

Para nosotras, lo rural es vivir en un pueblecito. Tiene menos de 4.000 habitantes. (Meritxell $^{3}, 39$, bellas artes/propietaria de una bodega, Cataluña)

En general, los hombres lo tienen mucho más claro:

Para mí, el campo tiene que ver con dos elementos: la actividad económica y el tamaño de la población (Juan José, 46, geógrafo/director de Instituto de Estudios Turísticos y profesor universitario, Cataluña).

Sin embargo, las mujeres tienden a definir el medio rural en contraposición al medio urbano, a pesar de que muchas actividades y formas de vida urbana se hayan extendido por todo el país:

A lo mejor lo definiría como lo que excluye lo urbano. Estoy segura de lo que es urbano, así pues, todo lo contrario (Elvira, 65, química y geógrafa/propietaria de un hotel rural, Cataluña).

Está claro que no todas las zonas rurales tienen el mismo grado de ruralidad y muchas mujeres lo destacan. Diferencian entre algunas zonas que son "más rurales" (aisladas, zonas de montaña, con una actividad agrícola muy tradicional) y las "otras" que están mejor conectadas en las que identifican una ruralidad que está desapareciendo.

Tal vez existen dos tipos de mundos rurales. Uno que está casi desapareciendo donde la gente todavía vive en granjas aisladas y dispersas... y otro en el que te puedes comportar como en la ciudad: te puedes vestir como en la ciudad y puedes ir a ver el Cirque du Soleil... Eso sí, necesitas un coche para todo (Maria Teresa, 57, propietaria de una tienda de artículos de papelería, Cataluña).

\footnotetext{
${ }^{3}$ Se han utilizado pseudónimos a fin de preservar el anonimato de todas las personas entrevistadas.
} 


\section{b) Sobre los idilios y los anti-idilios}

Las zonas rurales analizadas forman parte de la agricultura global, concebida como un espacio que responde a las condiciones de la interconexión global y a la interdependencia de las localidades rurales (Woods, 2007), y las mujeres que hemos entrevistado se encuentran, efectivamente, dentro de este contexto global. Así, la idea de las mujeres de la población rural está condicionada por los cambios discursivos y materiales del entorno mundial, así como por sus identidades y prácticas cotidianas. De esta manera, a menudo se cuestiona y se transgrede el concepto tradicional de idilio rural que se sustenta, con frecuencia, en las relaciones de género patriarcales (Little y Austin, 1996; Haugen et al. 2006).

\section{Un estilo de vida de calidad excepcional}

La idea de que el medio rural está vinculado a la calidad de vida es bastante omnipresente. Las mujeres se refieren al bienestar mental y físico, a la tranquilidad, a la buena alimentación y al vínculo con la naturaleza.

El campo es el lugar donde te sientas para ver cómo crece la vid... La vida rural es una vida sin prisas (Gema, 37, administrativa/emprendedora, Galicia).

Tal vez el tiempo... Es un modo de vida con distintas prioridades; disfrutas de las cosas (Mariña, 43, economista/propietaria de una casa de turismo rural, Galicia).

Sin embargo, las mujeres también son realistas y admiten los problemas derivados de esta forma de vida y desarrollan estrategias para minimizarlos:

Yo definiría el campo como el lugar perfecto para vivir, siempre que se cumplan unos requisitos mínimos. Por ejemplo, una mujer de 50 años de edad, que no sabe conducir y cuyos hijos ya se han ido de casa, no podría ni siquiera ir al médico (Marcela, 56, profesora de jardinería/gerente de un centro de información para mujeres, Galicia).

\section{El vínculo con la naturaleza}

Un gran número de mujeres mencionan que el paisaje rural permite una estrecha relación con la naturaleza. A veces sus comentarios reflejan una relación vivencial y espiritual con la naturaleza (Mies y Shiva, 1993) en particular en Galicia. Ponen énfasis en el hecho de pertenecer a la tierra, en quererla y cuidarla, lo que demuestra que, con frecuencia, muchas mujeres han encontrado en el paisaje (natural y construido) una fuente de fortaleza e identidad personal (Monk y Noorwood, 1987):

Esta es una manera de vivir, un sentido de pertenencia a la tierra, es un lazo... estamos genéticamente inclinados a sentir eso. Y para estar orgullosos de ser capaces de vivir de la tierra... para devolver lo que cogemos (Mariña, 43, economista/propietaria de una casa de turismo rural, Galicia). 
En algunas zonas como el Empordà, en Cataluña, se menciona directamente la belleza del paisaje. Este es el lugar donde muchas personas con mucho poder adquisitivo y político (principalmente de Barcelona) tienen una segunda residencia (primero en la Costa Brava y más tarde en el interior) y donde se ha producido un importante proceso de gentrificación (Solana, 2006). La población local ha interiorizado esta imagen reconstruida del Empordà como modelo de un idilio rural, literalmente ocupado por bienes inmuebles. Esta glorificación del paisaje no se produce en otras zonas rurales de igual belleza (siempre un concepto subjetivo), ya que hay poco turismo y no se ha construido idealmente como en el Empordà:

Diría que "la Conca" [su zona geográfica] es un área en gran parte desconocida, con un paisaje precioso. Algunas personas dicen que es como... la Toscana (en Italia). Estás cerca de las montañas y muy bien comunicado con todo... Es muy agradable para vivir, no hace ni frío ni calor (Aina, 53, farmacéutica y bióloga/propietaria de una farmacia, Cataluña).

\section{Relaciones próximas y también amigos lejanos}

El medio rural se concibe como un lugar donde las relaciones sociales son fáciles de construir.

Supongo que me gusta porque es similar a Irlanda [rural]; allí, siempre había alguien en casa, pasando por allí, entrando, yendo a por un café... La puerta siempre estaba abierta (Aileen, 43, filóloga/traductora, Cataluña).

Sin embargo, muchas de las mujeres tienen amigos en diferentes lugares que están bastante lejos y mantienen el contacto con ellos a través de Internet de forma rutinaria. Sin embargo, destacan que aprecian el contacto físico, ya que ayuda mucho a fortalecer las relaciones. No obstante también se observan los elementos negativos de la proximidad espacial de las personas conocidas. Las mujeres se quejan del exceso de control social, de los chismes, y el hecho de que muchos vecinos tienen una mentalidad cerrada.

Lo complicado es la gente. No puedes confiar completamente en nadie. Es un mundo muy cerrado y tú eres una extraña... y es muy fácil ignorar a la que acaba de llegar (Elisabet, 51, traducción e interpretación/gerente de un hostal rural, Cataluña).

\section{4. ¿QUÉ ES UNA MUJER RURAL?}

\section{a) Una mujer que (solamente) vive en el campo}

Algunas mujeres entrevistadas asocian el concepto de mujer rural a un modelo tradicional profundamente arraigado en la sociedad rural española. Este modelo implica un papel de domesticidad por parte de las mujeres y las obliga a cuidar de la familia y la explotación y las mantiene al margen de la toma de decisiones importantes. Pero este es el tipo de modelo que en el pasado ha expulsado a las mujeres de las zonas rurales y ha provocado un fuerte proceso de despoblación y masculinización: 
Una mujer rural era mi abuela. Se levantaba a las 6 de la mañana, trabajaba en el campo, comía muy poco, se encargaba de todas las tareas domésticas, lavaba a mano y trabajaba mucho. Sufrió mucho por la falta de dinero y ahorró tanto como pudo, por si se ponía enferma (Rita, 45, maestra/maestra de música, Cataluña).

Sin embargo, la mayoría de nuestras mujeres entrevistadas no encajan en este modelo, un modelo que hoy en día está limitado a zonas remotas o a mujeres de muy avanzada edad. De hecho, nuestras mujeres no se consideran rurales, a pesar de vivir en zonas rurales. Tienen recursos financieros suficientes y sobre todo educación, tienen iniciativas y un proyecto profesional. Por lo tanto, no se ven a ellas mismas ni diferente ni inferiores a las mujeres urbanas.

¿Si soy una mujer rural? Nunca me defino como rural, pero tal vez lo soy (Aileen, 43, filóloga/traductora, Cataluña).

Vivo en el campo, pero no soy rural (Natalia, 43, economista/propietaria de una casa, Galicia).

A ver, yo vivo y trabajo en el campo, pero yo no soy mi abuela... Tengo internet, puedo ir al cine, tengo un coche... Si me ves en otro contexto, me ves como una persona normal (Mariona, 29, ingeniera agrónoma y enóloga/agricultora, Cataluña).

\section{b) Trabajadora y decidida. Agencia y resistencia}

Consideran que las mujeres rurales (y ellas mismas) son personas trabajadoras y luchadoras y también agentes en la esfera pública, que es un logro importante. Sin embargo, la totalidad de su vida cotidiana refleja diferentes situaciones. La clase (en formas materiales y culturales) y el lugar tienen un gran efecto en su capacidad para superar las dificultades y aprovechar los beneficios de la vida rural.

Es muy difícil generalizar... porque veo mujeres inusuales... Destaco la iniciativa empresarial, el coraje personal y profesional, independientemente de las estructuras sociales de la región. Tienen la capacidad de crear proyectos, de innovar, sin tener en cuenta la estructura social (Juan José, 46, geógrafo/director de instituto de estudios turísticos y profesor universitario, Cataluña).

En los relatos muy a menudo aparecen las relaciones desiguales de poder, su aceptación y las tácticas que utilizan para superarlas. Sin embargo, a partir de las definiciones que dan las entrevistadas de "mujer rural" y de su propia imagen, las mujeres no se definen a ellas mismas ni a las mujeres rurales como esposas y madres, como se esperaría en el idilio rural. Sólo en pocos casos aparece la idea de la vida doméstica y la familia:

Hoy en día, hay mucha gente nueva, hay más apertura, y la mujer rural tiene más libertad que antes, pero sigue siendo un ama de casa. Le gusta cuidar a sus hijos, mantener la casa ordenada y tener una familia... (Ester, 54, maestra/agricultora, Galicia).

La definiría como una mujer fuerte, muy familiar y conectada con su entorno (más que una mujer de Barcelona) (Mònica, 36, trabajadora social/agente de igualdad de género, Cataluña). 


\section{c) Una mujer que puede usar un coche y conducirlo}

Poder tener un coche y saber conducir es una cuestión clave para poder vivir y trabajar en el campo. En las entrevistas se observa una alta movilidad para el trabajo, el ocio y la compra de bienes. Los viajes tienen, por lo general, diferentes propósitos:

Todo tipo de cosas... por ejemplo, puedo aprovechar para hacer las compras después de llevar al niño a la escuela, o voy a comprar antes si tengo que ir a los invernaderos por la mañana. Y cuando no hay otra opción, entonces voy a propósito (Míriam, de 52 años, licenciatura/agricultora, Galicia).

Las mujeres viajan a las ciudades para hacer grandes compras (semanales, mensuales), compras especializadas (ropa, calzado) y para servicios avanzados o para el ocio.

A Pira cuatro veces al día. Si vamos de compras, a Reus o Tarragona. Si tengo que ir a Barcelona, voy a Barcelona... (Marga, 54, bellas artes/propietaria de una bodega, Cataluña).

Voy a Lugo muy a menudo para hacer encargos, compras, para que nos proporcionen materiales. A veces vamos a Lalin... Vamos a Madrid por trabajo una vez al mes. Luego vamos a Coruña y a Santiago para el ocio (Natalia, 43, economista/propietaria de una casa de turismo rural, Galicia).

Las mujeres generalmente viajan en su coche o el de la familia y ello es posible por la espectacular mejora de las carreteras en España en las últimas décadas. En cambio el transporte público en las zonas rurales ha mejorado poco.

Todas las mujeres entrevistadas consideran que el coche es una condición sine qua non pero se quejan de la poca frecuencia del transporte público:

No tenemos comunicaciones de este tipo [bus regular]. Todo el mundo tiene un coche. (...) Si no, en taxi (Mar, 41, empresaria/ agricultora, Galicia).

Para la mayoría de las mujeres de nuestro estudio, la movilidad no es un inconveniente, pero es un verdadero problema para otras mujeres, mayores y jóvenes, que no están en la misma situación. Dicen:

Estas personas [las mujeres] mayores tienen cada vez más dificultades para ir de compras. Van a pie. No han tenido que conducir nunca. Ellas siempre han estado aquí... Debería haber un servicio semanal que las lleve a comprar (Abril, 36, turismo/técnica de turismo, Cataluña).

La movilidad virtual también es muy importante. De esta manera, nuestras mujeres establecen múltiples relaciones sociales a nivel profesional y personal en muchos lugares (en España y en el extranjero) y esto es algo que valoran en gran manera, ya que, de esta manera, no se sienten aisladas.

Hablamos por email con mis amigos enólogos que se encuentran dispersos por todo el mundo (La Rioja, Galicia...). Tengo dos cuentas y utilizo el Hotmail con mis amigos (Mariona, 29, ingeniera agrónoma y enóloga/agricultora, Cataluña). 


\section{CONCLUSIONES}

Queda claro que las mujeres en su discurso sobre la ruralidad perciben la vida cotidiana del mundo rural desde una clara perspectiva de género. Desde su posicionalidad, es muy difícil definir qué es "el Medio Rural” y sobre todo qué es "la Mujer Rural”. Por eso tienden a etiquetar como "rural" todo lo que es "no urbano". Sin embargo, reconocen que en el medio rural de hoy se encuentran muchos elementos "urbanos" (bienes materiales, educación, etc.). De este discurso sobre lo rural se desprende que la dualidad entre urbano/rural como categoría de análisis ha quedado desfasada e introduce un desafío en el significado de los dos conceptos, que deben entenderse más como procesos que como entidades rígidas. En sus discursos las mujeres crean, por un lado, una construcción social negativa de la ciudad (muy relacionada con el estrés) y, por el otro, una construcción social más positiva de las mujeres de zonas urbanas, en particular porque las ven como realmente independientes. En cualquier caso, la idea de cambio está siempre presente en sus narraciones y las mujeres hacen hincapié en las enormes diferencias entre su generación, y las de sus madres y abuelas.

Las mujeres claramente relacionan el medio rural con la calidad de vida. Para ellas la vida rural está asociada a un entorno tranquilo, a relaciones sociales más cercanas y a un contacto directo con la naturaleza. Este hecho refleja claramente su posición de clase. En efecto, la muestra incluye sólo a mujeres profesionales que han decidido desarrollar su proyecto personal y profesional en el medio rural. Esta posición de clase implica disponer de recursos materiales e inmateriales para poder disfrutar de esta calidad de vida, y para minimizar los efectos negativos del medio rural, como el desempleo, la falta de incentivos o la exclusión social.

Las mujeres no se ven a ellas mismas como "rurales" como lo son las mujeres tradicionales. Los primeros adjetivos que utilizan para definir a las mujeres rurales (incluidas ellas mismas) son trabajadoras y luchadoras, y consideran que la movilidad es una cuestión clave y una condición previa que les permite permanecer en el campo y desarrollar sus proyectos personales. Curiosamente, destacan sus habilidades personales en vez de su papel social. Por lo tanto, no definen a las mujeres rurales (incluidas ellas mismas) como esposas y madres, como se esperaría en la construcción social del idilio rural tradicional. Sin embargo, en su vida diaria valoran sus roles de género como madres y cuidadoras de la familia a pesar de que creen que no les impiden tener su propio proyecto de vida. En los relatos se observa que la distribución del trabajo dentro del hogar no está equilibrada según el género y, de hecho, las mujeres transgreden sólo ligeramente el rol doméstico que se les asigna socialmente. Muchos de sus problemas de la vida cotidiana no surgen del hecho de vivir en zonas rurales, sino de su capacidad/incapacidad de negociar su rol social con los hombres. Las ideas de "subordinación” y "sacrificio", aunque todavía están presentes, se han convertido eufemísticamente en la "capacidad de trabajo", en referencia a la carga doble o triple de las mujeres.

Las narraciones de los hombres se diferencian ligeramente de las de las mujeres. Las principales diferencias son que los hombres no hacen referencia a las relaciones sociales cuando definen la familia y el medio rural, y que no asocian el medio rural con la naturaleza. Las narraciones de los hombres también ponen énfasis en la definición de las mujeres rurales como muy trabajadoras y emprendedoras (igual que señalan las mujeres), pero 
en algunas zonas (principalmente en Galicia) se percibe que las mujeres son demasiado dependientes de los hombres.

En definitiva, la percepción que las mujeres tienen de la ruralidad y de sí mismas como "mujeres rurales" representa un documento insustituible para el estudio de la vida cotidiana de nuestros tiempos en el medio rural.

\section{BIBLIOGRAFÍA}

ALDOMÀ, I. (dir.) (2009): Atles de la nova ruralitat, Lleida, Fundació Món Rural.

BAYLINA, M., BERG, N.G. (2010): "Selling the countryside: Representations of rurality in Norway and Spain", European Urban and Regional Studies, 17 (3), 277-292.

BAYONA, J., GIL, F. (2013): "Is foreign immigration the solution to rural depopulation? The case of Catalonia (1996-2009)", Sociologia Ruralis, 53 (1), 26-50.

BULLER, H., HOGGART, K. (eds.) (2004): Women in the European countryside, Aldershot, Ashgate, $162 \mathrm{p}$.

CAMARERO, L. (2009): “¿Por qué hay menos mujeres en las áreas rurales?, Agricultura familiar en España 2009" en: Anuario de la Fundación de Estudios Rurales, Unión de Pequeños Agricultores y Ganaderos, 86-90.

CAMARERO, L., SAMPEDRO, R., VICENTE-MAZARIEGOS, J.L. (1991): Mujer y ruralidad. El círculo quebrado, Madrid, Ministerio de Asuntos Sociales / Instituto de la Mujer, 237 p.

CARBÓ, M., BAYLINA, M., GARCIA RAMON, M.D. (2013): "Women's ventures in a rural context: livelihood and identity", Hagar. Studies in Culture, Polities and Identities, 11 (1), 100-120.

FADEMUR (2009): “Mujeres rurales en España, Agricultura familiar en España 2009” en: Anuario de la Fundación de Estudios Rurales, Unión de Pequeños Agricultores y Ganaderos, 61-77.

GARCÍA, B. (2011): Ruralidad emergente, posibilidades y retos, Madrid, Ministerio de Medio Ambiente, Medio Rural y Marino, 538 p.

GARCÍA, A., SÁNCHEZ, D. (2005): "La población rural en Catalunya: entre el declive y la revitalización”, Cuadernos Geográficos, 36, 387-407.

GARCIA RAMON, M.D, CRUZ, J., SALAMAÑA, I., VILLARINO, M. (1994): Mujer y agricultura en España. Género, trabajo y contexto regional, Vilassar de Mar, Oikos-Tau, 179 p.

GARCIA RAMON, M.D., BAYLINA, M. (eds.) (2000): El nuevo papel de las mujeres en el desarrollo rural, Vilassar de Mar, Oikos-Tau, $336 \mathrm{p}$.

GOVERDE, H., DE HAAN, H., BAYLINA, M. (eds.) (2004): Power and gender in European Rural Development, Aldershot, Ashgate.

GUIRADO, C. (2010): Tornant a la muntanya. Migració, ruralitat i canvi social al Pirineu català. El cas del Pallars Sobirà. Tesis doctoral. Departament de Geografia, Universitat Autònoma de Barcelona, $693 \mathrm{p}$.

HALFACREE, K. (2006): "Rural space: constructing a three-fold architecture", Cloke, P., Marsden, T., Mooney, P. (eds.), Handbook of rural studies, London, Sage, 63-90.

HALFACREE, K. (2007): "Still surprises in store: Revisiting the ordinary in rural geography", Documents d'Anàlisi Geogràfica, 50, 87-103.

HAUGEN, M., VILLA, M. (2006): "Rural idylls or boring places?" en: Bock, B., Shorthall, S. (eds.), Rural gender relations. Issues and case studies, Wallingford, Cabi Publishing, 181-195.

HOGGART, K., PANIAGUA, A. (2002): “The restructuring of rural Spain?", Journal of Rural Studies, 17, 63-80.

IDESCAT (Institut d'Estadística de Catalunya) (2012): Estadístiques població ocupada, Barcelona, Generalitat de Catalunya.

LITTLE, J. (2001): Gender and rural geography. Identity, sexuality and power in the countryside, Harlow, Prentice Hall, 213 p. 
LITTLE, J., AUSTIN, P. (1996): "Women and the rural idyll”, Journal of Rural Studies, 12, 101-111. MIES, M., SHIVA, V. (1993): Ecofeminism, London, Zed Books, 336 p.

MINISTERIO DE MEDIO AMBIENTE Y MEDIO RURAL Y MARINO (MARM) (2011): Diagnóstico de la igualdad de género en el medio rural, Madrid, Gobierno de España, 338 p.

MORÉN, R., SOLANA, M. (2006): "La immigració en àrees rurals i petites ciutats d'Espanya. Un estat de la questió", Documents d'Anàlisi Geogràfica, 47, 141-155.

MONK, J., NORWOOD, V. (1987): The desert is no lady. Sowthwestern landscapes in women's writing and art, Tucson, University of Arizona Press, $281 \mathrm{p}$.

SABATÉ, A. (1992): "La participación de las mujeres en la dinámica social de zonas rurales desfavorecidas" en: Desarrollo local y Medio Ambiente en zonas desfavorecidas, Madrid, Ministerio de Obras Públicas y Transportes, 123-138.

SACHS, C. (1996): Gendered fields. Rural women, agriculture and environment, Boulder, Westview Press, $205 \mathrm{p}$.

SAMPEDRO, R. (2009): "Escuchando la voz de las mujeres" en: Agricultura familiar en España 2009, Anuario de la Fundación de Estudios Rurales, Unión de Pequeños Agricultores y Ganaderos, 92-102.

SOLANA, Miguel (2006): "Nuevas dinámicas migratorias en los espacios rurales: vivienda, cambio social y procesos de elitización. El caso del Empordanet (Girona)", Ager, 5, 57-87.

WOODS, M. (2007): "Engaging the global countryside: globalization, hybridity and the reconstitution of rural place", Progress in Human Geography, 31, 485-507.

WOODS, M. (2011): "Rural Europe and the world: Globalization and rural development" (editorial), European Countryside, 3, 153-163. 
